\title{
Resistência varietal de milho doce crioulo a Spodoptera frugiperda (J.E. Smith) (Lepidoptera: Noctuidae)
}

Varietal resistance of creole Sweet corn to Spodoptera frugiperda (J.E. Smith) (Lepidoptera: Noctuidae)

Resistencia varietal del maíz dulce criollo a Spodoptera frugiperda (J.E. Smith) (Lepidoptera: Noctuidae)

\section{Resumo}

A lagarta-do-cartucho Spodoptera frugiperda (J. E. Smith) (Lepidoptera: Noctuidae) é praga-chave na cultura do milho doce (Zea mays L.). Seu manejo é baseado no uso de inseticidas de amplo espectro, causando impactos ambientais e à saúde humana. Neste sentido, o emprego de variedades resistentes ao ataque da praga é uma estratégia de manejo menos impactante. Este trabalho avaliou o desempenho de variedades de milho doce ao ataque de $S$. frugiperda. O experimento foi conduzido em condições de campo, em blocos completamente casualizados com quatro repetições para cada uma das vinte variedades de milho doce testadas, sendo 19 crioulas e uma fornecida pelo Instituto Agronômico de Campinas. Os danos de $S$. frugiperda foram avaliados conforme escala visual de notas e o monitoramento da flutuação populacional foi realizado por meio de armadilhas Delta com feromônio sintético. Houve diferença significativa quanto ao nível de danos nas amostragens realizadas aos 42, 63, 70 e 77 dias após a semeadura. Dentre as variedades testadas, 66,7 \% apresentaram nível de dano baixo e 33,3\% apresentaram nível médio. A flutuação populacional de machos de $S$. frugiperda se manteve abaixo do nível de dano econômico ao longo de toda a condução do experimento. Uma possível resistência das variedades avaliadas associada a fatores ambientais e pulverização com inseticidas pode ter contribuído para o comportamento e para os baixos níveis de danos causados por $S$. frugiperda.

Palavras-chave: Lagarta-do-cartucho; Manejo integrado de pragas; Zea mays L.

\begin{abstract}
The fall armyworm Spodoptera frugiperda (J. E. Smith) (Lepidoptera: Noctuidae) is considered a key pest in the conditions of cultivation of sweet corn (Zea mays L.). It's management is based on the use of broad-spectrum insecticides, whose use results in great environmental and human health impacts. In this sense, the use of varieties that are resistant to pest attack emerges as a less impactful management strategy. This study analyzed the performance of creole and commercial sweet corn varieties against the attack of S. frugiperda. The experiment was conducted under field conditions in randomized complete block design with four replications for each of the twenty sweet corn varieties tested, 19 of which were creole and one provided by the Instituto Agronômico de Campinas. The damages of $S$. frugiperda were evaluated according to the visual scale of notes and the monitoring of population fluctuation with use of Delta traps with synthetic pheromone. There was a significant difference in the level of damage at 42, 63, 70 and 77 days after sowing. Among the varieties tested, $66.7 \%$ had a low level of damage and $33.3 \%$ had a medium level. The population fluctuation of males of $S$. frugiperda remained below the level of economic damage throughout the study period. A possible resistance of the evaluated varieties associated with environmental factors and spraying with insecticides may have contributed to the behavior for the low level of damage caused by S. frugiperda.
\end{abstract}

Keywords: Fall armyworm; Integrated pest management; Zea mays L. 


\begin{abstract}
Resumen
La oruga de cartucho Spodoptera frugiperda (J. E. Smith) (Lepidoptera: Noctuidae) es una plaga clave en el cultivo de maíz dulce (Zea mays L.). Su manejo se basa en el uso de insecticidas de amplio espectro, que provocan impactos ambientales y em la salud humana. em este sentido, el uso de variedades resistentes al ataque de la plaga es una estrategia de manejo de menor impacto. Este trabajo evaluó el comportamiento de variedades de maíz dulce al ataque de $S$. frugiperda. El experimento se realizó en condiciones de campo en bloques completamente al azar con cuatro repeticiones para cada una de las veinte variedades de maíz dulce probadas, 19 de las cuales fueron criollas y una proporcionada por el Instituto Agronômico de Campinas. Los daños de $S$. frugiperda se evaluaron según la escala visual de notas y el seguimiento de la fluctuación poblacional se realizó mediante trampas Delta con feromona sintética. Hubo una diferencia significativa en el nivel de daño en las muestras tomadas a los 42, 63, 70 y 77 días después de la siembra. Entre las variedades probadas, el 66,7\% tuvo un nivel de daño bajo y el 33,3\% tuvo un nivel medio. La fluctuación de la población de machos de $S$. frugiperda permaneció por debajo del nivel de daño económico durante la realización del experimento. Una posible resistencia de las variedades evaluadas asociada a factores ambientales y la fumigación con insecticidas pudo haber contribuido al comportamiento por los bajos niveles de daño causado por S. frugiperda.
\end{abstract}

Palabras clave: Oruga de cartucho; Manejo integrado de plagas; Zea mays L.

\title{
1. Introdução
}

O milho (Zea mays L.) é amplamente cultivado no Brasil. Dentre as variedades produzidas, o milho doce se destaca por ser amplamente empregado para o consumo humano, sendo utilizado na produção de enlatados e consumido in natura, desidratado, congelado, como minimilho (colhido antes da polinização) ou mesmo utilizado como silagem (Teixeira et al., 2001; Bordallo et al., 2005; Oliveira Jr. et al., 2006). Atualmente existem variedades comerciais de milho doce, entretanto agricultores familiares mantêm variedades crioulas cujos principais destinos da produção são a troca de sementes, o consumo in natura ou o uso em pratos culinários típicos (De Andrade et al., 2002; Souza, 2015).

A ocorrência de pragas é um dos principais problemas na cultura do milho doce. Entre as pragas chave destaca-se Spodoptera frugiperda (J. E. Smith) (Lepidoptera: Noctuidae), conhecida como lagarta-do-cartucho ou lagarta-militar. As larvas atacam plantas de milho durante todo o ciclo da cultura, causando prejuízos econômicos (Cruz, Figueiredo \& Da Silva, 2010). A lagarta se alimenta do cartucho do milho causando danos tanto no colmo (coração morto) como em folhas, sendo mais prejudicial quando o ataque ocorre em plantas jovens (Cruz, 1995; Casmuz et al., 2010). Além disso, é possível que variedades de milho doce apresentem menor resistência ao ataque de pragas quando comparado ao milho convencional, sendo isso atribuído aos maiores teores de açúcares presentes neste tipo de milho (Oliveira Jr. et al., 2006).

O uso de inseticidas com amplo espectro de ação é uma das principais estratégias para controle de $S$. frugiperda (DiezRodríguez \& Omoto, 2001; León-García et al., 2012; Fazolin et al., 2015). Entretanto, o uso abusivo de inseticidas pode causar efeitos indesejados como a seleção de populações resistentes, contaminação humana e ambiental, além da eliminação de insetos benéficos (Ribas \& Matsumura, 2009; Bohner, Araújo \& Nishijima, 2013; Bolzan, 2019). Nesse contexto, se faz importante a busca por estratégias menos impactantes para o manejo de $S$. frugiperda em milho doce.

O emprego de variedades de milho resistentes ao ataque de pragas é uma estratégia importante para o manejo de $S$. frugiperda. Plantas resistentes podem afetar o desenvolvimento e comportamento de insetos, reduzindo o uso de inseticidas (Aoyama \& Labinas, 2012). Além disso, podem apresentar as mesmas vantagens do milho geneticamente modificados para o controle de insetos, que receberam genes de Bacillus thuringiensis (Bt), resistentes a S. frugiperda, sem a necessidade de conhecimentos específicos e técnicas adicionais para sua utilização (Ota et al., 2011). Nesse sentido, o emprego de variedades de milho doce resistentes ao ataque de $S$. frugiperda pode proporcionar a redução das aplicações de inseticidas.

Apesar dos investimentos em tecnologias que reduzam os danos causados por insetos na cultura do milho, são poucos os trabalhos direcionados para o manejo de pragas em milho doce. A utilização de variedades crioulas selecionadas por agricultores pode fornecer material vegetal que apresente resistência a $S$. frugiperda, além de favorecer a conservação da biodiversidade e a 
autonomia do produtor rural familiar (Catão et al., 2010). Nesse contexto, este estudo avaliou a resistência de 19 variedades crioulas e uma variedade comercial de milho doce ao ataque de S. frugiperda.

\section{Material e Métodos}

\subsection{Características da área}

O estudo foi conduzido de outubro de 2019 a janeiro de 2020 na Fazenda Experimental Ressacada da Universidade Federal de Santa Catarina ( $27^{\circ} 41^{\prime} 06.28^{\prime \prime}$ S; $48^{\circ} 32^{\prime} 38.81^{\prime \prime}$ O). O clima é classificado como Cfa, com umidade constante e verões quentes, temperatura anual média de $21^{\circ} \mathrm{C}$, precipitação anual acumulada de aproximadamente $1.700 \mathrm{~mm}$, sem estação seca e umidade relativa aproximada de 82\% (Alvares et al., 2013). O solo é classificado como Neossolo Quartzarênico Hidromórfico Típico, preponderantemente constituído de areia, apresentando altos teores de matéria orgânica e lençol freático próximo à superfície (Dos Santos et al., 2018).

\subsection{Material vegetal}

Foram avaliadas 20 variedades de milho doce crioulo, sendo 19 variedades provenientes de comunidades rurais dos municípios de Anchieta ( $26^{\circ} 32^{\prime} 4^{\prime \prime} \mathrm{S}$; $53^{\circ} 20^{\prime} 11^{\prime \prime}$ O) e Guaraciaba (20 $33^{\prime} 28^{\prime \prime} \mathrm{S} ; 43^{\circ} 0^{\prime} 22^{\prime \prime}$ O) no extremo oeste de Santa Catarina e uma variedade fornecida pelo Instituto Agronômico de Campinas (IAC). A caracterização fenotípica das variedades utilizadas neste estudo são descritas por Parentoni et al. (1990) e Souza (2015). Os nomes das variedades correspondem aos códigos do banco de germoplasma do laboratório NEABio da UFSC. Dentre as variedades testadas, o milho doce Cubano desenvolvido pelo IAC e as variedades crioulas 2029A, 2255A, 2276A, 319A e 741B do extremo oeste catarinense são parentais enquanto os demais tratamentos são compostos pelos respectivos híbridos intervarietais dos genitores (Tabela 1).

Tabela 1. Variedades de milho doce genitores e híbridos obtidos por cruzamentos.

\begin{tabular}{ccc}
\hline Genitores & Híbridos & Híbridos \\
\hline Cubano (IAC) & Cubano $\times 2029 \mathrm{~A}$ & $741 \mathrm{~B} \times 319 \mathrm{~A}$ \\
$741 \mathrm{~B}$ & Cubano $\times 2255 \mathrm{~A}$ & $2255 \mathrm{~A} \times 2029 \mathrm{~A}$ \\
$2255 \mathrm{~A}$ & Cubano $\times 2276 \mathrm{~A}$ & $2255 \mathrm{~A} \times 2276 \mathrm{~A}$ \\
$2276 \mathrm{~A}$ & Cubano $\times 319 \mathrm{~A}$ & $2255 \mathrm{~A} \times 319 \mathrm{~A}$ \\
$2029 \mathrm{~A}$ & Cubano $\times 741 \mathrm{~B}$ & $2255 \mathrm{~A} \times 741 \mathrm{~B}$ \\
$319 \mathrm{~A}$ & $741 \mathrm{~B} \times 2029 \mathrm{~A}$ & $2276 \mathrm{~A} \times 2029 \mathrm{~A}$ \\
---- & $741 \mathrm{~B} \times 2276 \mathrm{~A}$ & $2029 \mathrm{~A} \times 319 \mathrm{~A}$ \\
\hline
\end{tabular}

Fonte: Autores.

\subsection{Plantio e tratos culturais}

O preparo da área foi através de aração e gradagem com incorporação de adubo. Para adubação foi empregado $12 \mathrm{Kg}$ de ureia, 24,5 Kg de superfosfato triplo e 16,5 Kg de KCl, sendo baseada em análise de solo e de acordo com o Manual de adubação e de calagem para os estados do Rio Grande do Sul e Santa Catarina (Tedesco et al., 2004). A semeadura foi efetuada manualmente em linhas demarcadas pela passagem dos discos de corte do implemento acoplado ao trator. As variedades de milho doce foram plantadas em blocos completamente casualizados, empregando-se quatro repetições. Cada repetição foi formada por três linhas com 15 plantas no espaçamento de 1,0 m entre linhas e 0,2 m entre plantas, totalizando 45 plantas por parcela. Aos 38 dias após a semeadura (DAS) realizou-se a adubação de cobertura com ureia (5 Kg). A aplicação de ureia foi baseada em análise de solo e de acordo com o Tedesco et al. (2004). Em virtude da alta infestação inicial de lagartas de $S$. 
frugiperda, foram realizadas aplicações de inseticidas aos 34 DAS com inseticida Keshet ${ }^{\circledR} 25 \mathrm{EC}$ (piretroide) e aos 51 DAS com inseticida Tracer $^{\circledR}$ (espinosina). O uso destes inseticidas foi baseado em avaliações visuais de danos causados pelas larvas, conforme escala de Davis (1989). Se durante o monitoramento $20 \%$ ou mais de plantas apresentarem nota igual ou superior a três (folhas com pequenas lesões circulares e algumas pequenas lesões alongadas, forma de retângulo, nas folhas do cartucho do milho) o controle químico do inseto deve ser realizado.

\subsection{Avaliações de danos foliares}

Os danos foliares causados por larvas de S. frugiperda foram avaliados semanalmente, conforme metodologia descrita por Barcelos \& Angelini (2018). Em cada avaliação, dez plantas foram escolhidas aleatoriamente dentro de cada tratamento desde o estádio vegetativo V3 até o pendoamento em VT. Em virtude do pendoamento desuniforme entre as variedades, a época de avaliação dos danos no estádio VT variou ao longo da condução do experimento. As notas atribuídas às lesões baseiam-se no sistema de classificação proposto por Davis, Williams \& Wiseman (1989), variando o nível de dano de 1 (altamente resistente) a 9 (altamente susceptível) (Tabela 2).

Tabela 2. Escala visual de notas para danos de S. frugiperda em milho.

\begin{tabular}{|c|c|c|}
\hline Nível de dano & Escala & Caracterização \\
\hline \multirow{3}{*}{ Baixo } & 1 & Nenhum dano até três lesões muito pequenas nas folhas do cartucho. \\
\hline & 2 & Lesões muito pequenas e circulares nas folhas do cartucho. \\
\hline & 3 & $\begin{array}{l}\text { Pequenas lesões circulares e algumas pequenas lesões alongadas (formato de retângulo) nas folhas } \\
\text { do cartucho. }\end{array}$ \\
\hline \multirow{3}{*}{ Médio } & 4 & $\begin{array}{l}\text { De } 4 \text { a } 7 \text { lesões alongadas, pequenas ou médias de } 1,3 \text { a } 2,5 \mathrm{~cm} \text {, em algumas folhas do cartucho e } \\
\text { folhas expandidas. }\end{array}$ \\
\hline & 5 & $\begin{array}{l}\text { De } 4 \text { a } 7 \text { lesões alongadas grandes, maiores que } 2,5 \mathrm{~cm} \text { de comprimento em algumas folhas do } \\
\text { cartucho e folhas expandidas e/ou alguns furos pequenos e médios de formato uniforme e irregular } \\
\text { (membrana consumida) no cartucho e nas folhas expandidas. }\end{array}$ \\
\hline & 6 & $\begin{array}{l}\text { De } 4 \text { a } 7 \text { lesões alongadas em várias folhas do cartucho e expandidas e/ou vários furos uniformes } \\
\text { a irregulares nas folhas do cartucho e expandidas. }\end{array}$ \\
\hline \multirow{3}{*}{ Alto } & 7 & $\begin{array}{l}8 \text { ou mais lesões alongadas de todos os tamanhos em várias folhas do cartucho, além de furos de } \\
\text { formato uniforme a irregular nas folhas do cartucho e expandidas. }\end{array}$ \\
\hline & 8 & $\begin{array}{l}8 \text { ou mais lesões alongadas de todos os tamanhos presentes na maioria das folhas do cartucho, } \\
\text { além de muitos furos médios a grandes de formato uniforme a irregular nas folhas do cartucho e } \\
\text { expandidas. }\end{array}$ \\
\hline & 9 & Cartuchos e folhas expandidas quase totalmente destruídos. \\
\hline
\end{tabular}

Fonte: Adaptado de Davis, Williams e Wiseman (1989).

\subsection{Flutuação populacional de S. frugiperda}

O monitoramento de machos adultos de S. frugiperda foi realizado semanalmente por meio de duas armadilhas tipo Delta instaladas 24 DAS e verificadas semanalmente até o estádio VT (pendoamento), totalizando oito avaliações. As armadilhas foram posicionadas a noroeste e sudeste da área experimental a 1,5 m de altura, havendo troca de septos de feromônios sexuais sintéticos Bio Spodoptera $^{\circledR}$ a cada 30 dias e das placas adesivas conforme a necessidade. 


\subsection{Análises estatísticas}

Os danos foliares causados por larvas de S. frugiperda foram submetidos ao teste de normalidade (Shapiro \& Wilk, 1965) e à análise de variância (One way ANOVA). As médias foram comparadas pelo teste de Tukey $(\mathrm{p}=0,05)$ por meio do software Minitab 18.0 (Minitab Inc. State College, Pennsylvania).

\section{Resultados e Discussão}

As variedades de milho doce apresentaram diferenças significativas nos níveis de danos foliares em avaliações realizadas aos 42, 63, 70 e 77 DAS (Tabela 3). De acordo com a escala visual de notas, as variedades crioulas de milho doce apresentaram um nível baixo de dano em 66,7\% das avaliações (notas 1, 2 e 3) e em apenas 33,3\% o nível de dano foi considerado médio (notas 4, 5 e 6). Além de uma possível resistência natural das variedades, fatores ambientais e o uso de inseticidas podem ter contribuído para a redução da população de S. frugiperda a campo (Cruz, 1995), o que colaborou para que os danos causados por lagartas tenham sido classificados como baixos na maioria das avaliações. As aplicações de inseticidas sob as quais as variedades foram submetidas indicam que este fator tenha expressado efeitos significativos sobre as notas identificadas nas avaliações. No entanto, os resultados obtidos apresentam relevância, uma vez que todas as variedades foram sujeitas à mesma condição.

A elevada variabilidade genética é um fator importante para a resistência natural de milhos crioulos, pois pode favorecer o desenvolvimento de mecanismos de defesa que afetam a alimentação e a oviposição, além de causar antibiose em insetos desfolhadores. A não preferência alimentar ocorre em milho crioulo para S. frugiperda (Nogueira, 2015). Essa característica pode ser atribuída a composição de lipídios cuticulares com altas concentrações de n-alcanos, repelentes da alimentação (Yang et al., 1993) ou a presença de moduladores da alimentação (Johnson, Snook \& Wiseman, 2002; Simmonds, 2003). 
Research, Society and Development, v. 10, n. 13, e411101321309, 2021

(CC BY 4.0) | ISSN 2525-3409 | DOI: http://dx.doi.org/10.33448/rsd-v10i13.21309

Tabela 3. Danos foliares causados por larvas S. frugiperda (Lepidoptera: Noctuidae) em vinte variedades de milho doce.

\begin{tabular}{|c|c|c|c|c|c|c|c|c|c|}
\hline \multirow{2}{*}{ Tratamento } & \multicolumn{9}{|c|}{ Dias Após a Semeadura (DAS) } \\
\hline & 21 & 28 & 35 & 42 & 49 & 56 & 63 & 70 & 77 \\
\hline Cubano & $3,4( \pm 0,16)$ & $3,5( \pm 0,56)$ & $5,3( \pm 0,56)$ & $4,3( \pm 0,67) a b$ & $4,8( \pm 0,51)$ & $3,7( \pm 0,56)$ & $3,9( \pm 0,75)$ abc & $2,8( \pm 0,33) \mathrm{a}$ & ----- \\
\hline Cubano $\times 2029 A$ & $2,9( \pm 0,43)$ & $3,8( \pm 0,33)$ & $5,0( \pm 0,52)$ & $2,5( \pm 0,45) a b$ & $3,4( \pm 0,62)$ & $4,2( \pm 0,61)$ & $3,5( \pm 0,69) a b c$ & $4,9( \pm 0,57) \mathrm{abc}$ & $3,7( \pm 0,47)$ abc \\
\hline Cubano $\times 2255 \mathrm{~A}$ & $2,6( \pm 0,27)$ & $3,5( \pm 0,27)$ & $5,2( \pm 0,42)$ & $3,0( \pm 0,39) \mathrm{ab}$ & $4,2( \pm 0,65)$ & $3,4( \pm 0,56)$ & $3,0( \pm 0,42) \mathrm{abc}$ & $3,5( \pm 0,31) a b$ & ----- \\
\hline Cubano $\times 2276 \mathrm{~A}$ & $1,8( \pm 0,29)$ & $4,0( \pm 0,58)$ & $3,9( \pm 0,80)$ & $3,4( \pm 0,54) a b$ & $3,4( \pm 0,81)$ & $3,4( \pm 0,69)$ & $3,8( \pm 0,70) a b c$ & $4,3( \pm 0,45)$ abc & $3,7( \pm 0,42)$ abc \\
\hline Cubano $\times 319 \mathrm{~A}$ & $3,1( \pm 0,35)$ & $4,2( \pm 0,73)$ & $4,7( \pm 0,60)$ & $3,4( \pm 0,40) a b$ & $4,3( \pm 0,70)$ & $4,8( \pm 0,51)$ & $4,3( \pm 0,50) \mathrm{abc}$ & $3,5( \pm 0,48) a b$ & ----- \\
\hline Cubano $\times 741 B$ & $2,3( \pm 0,45)$ & $4,4( \pm 0,37)$ & $3,6( \pm 0,37)$ & $5,1( \pm 0,77) a b$ & $5,4( \pm 0,81)$ & $4,4( \pm 0,81)$ & $3,9( \pm 0,62) a b c$ & $5,2( \pm 0,49) a b c$ & $4,0( \pm 0,71)$ abc \\
\hline $741 B$ & $2,9( \pm 0,31)$ & $4,7( \pm 0,84)$ & $5,6( \pm 1,01)$ & $2,4( \pm 0,69) \mathrm{a}$ & $3,2( \pm 0,57)$ & $3,9( \pm 0,35)$ & $5,1( \pm 0,60) b c$ & $6,7( \pm 0,36) c$ & ----- \\
\hline $741 B \times 2029 A$ & $2,7( \pm 0,37)$ & $3,6( \pm 0,65)$ & $5,3( \pm 0,76)$ & $2,7( \pm 0,68) a b$ & $3,6( \pm 0,69)$ & $4,1( \pm 0,41)$ & $5,5( \pm 0,54) \mathrm{c}$ & $5,1( \pm 0,78)$ abc & $3,9( \pm 0,48)$ abc \\
\hline $741 \mathrm{~B} \times 2276 \mathrm{~A}$ & $3,1( \pm 0,38)$ & $4,3( \pm 0,50)$ & $5,7( \pm 0,78)$ & $3,9( \pm 0,81) a b$ & $4,2( \pm 0,83)$ & $4,7( \pm 0,52)$ & $3,4( \pm 0,37) a b c$ & $3,4( \pm 0,56) a b$ & $3,6( \pm 0,67) a b$ \\
\hline $741 \mathrm{~B} \times 319 \mathrm{~A}$ & $2,2( \pm 0,42)$ & $3,3( \pm 0,33)$ & $3,6( \pm 0,70)$ & $2,9( \pm 0,31) a b$ & $2,6( \pm 0,58)$ & $3,3( \pm 0,52)$ & $2,3( \pm 0,42) a b$ & $3,6( \pm 0,52) a b$ & $3,0( \pm 0,33) \mathrm{a}$ \\
\hline $2255 \mathrm{~A}$ & $3,2( \pm 0,36)$ & $3,7( \pm 0,54)$ & $3,8( \pm 0,81)$ & $3,7( \pm 0,60) a b$ & $4,3( \pm 0,72)$ & $4,6( \pm 0,34)$ & $5,5( \pm 0,52) \mathrm{c}$ & $5,1( \pm 0,64)$ abc & $6,5( \pm 0,65) c$ \\
\hline $2255 \mathrm{~A} \times 2029 \mathrm{~A}$ & $2,9( \pm 0,28)$ & $2,9( \pm 0,28)$ & $4,5( \pm 0,65)$ & $3,5( \pm 0,60) a b$ & $4,6( \pm 0,65)$ & $3,6( \pm 0,52)$ & $4,2( \pm 0,29) \mathrm{abc}$ & $5,1( \pm 0,46) a b c$ & $3,8( \pm 0,49)$ abc \\
\hline $2255 \mathrm{~A} \times 2276 \mathrm{~A}$ & $2,4( \pm 0,31)$ & $2,5( \pm 0,43)$ & $5,1( \pm 0,91)$ & $5,7( \pm 0,73) b$ & $4,0( \pm 0,82)$ & $3,2( \pm 0,53)$ & $5,1( \pm 0,69) a b c$ & $6,3( \pm 0,26) c$ & $5,9( \pm 0,95) b c$ \\
\hline $2255 \mathrm{~A} \times 319 \mathrm{~A}$ & $2,3( \pm 0,37)$ & $2,9( \pm 0,41)$ & $5,2( \pm 0,85)$ & $3,2( \pm 0,66) a b$ & $3,7( \pm 0,65)$ & $2,9( \pm 0,53)$ & $2,2( \pm 0,29) \mathrm{a}$ & $3,6( \pm 0,72) a b$ & $2,9( \pm 0,41) \mathrm{a}$ \\
\hline $2255 \mathrm{~A} \times 741 \mathrm{~B}$ & $3,0( \pm 0,26)$ & $3,1( \pm 0,74)$ & $4,0( \pm 0,60)$ & $3,5( \pm 0,89) a b$ & $3,6( \pm 0,60)$ & $3,1( \pm 0,60)$ & $2,7( \pm 0,50) a b c$ & $4,7( \pm 0,47) a b c$ & $2,9( \pm 0,43) \mathrm{a}$ \\
\hline $2276 \mathrm{~A}$ & $2,8( \pm, 036)$ & $3,6( \pm 0,52)$ & $3,5( \pm 0,73)$ & $3,9( \pm 1,05) \mathrm{ab}$ & $3,5( \pm 0,73)$ & $3,5( \pm 0,52)$ & $3,2( \pm 0,80)$ abc & $5,2( \pm 0,68) a b c$ & $5,4( \pm 0,86)$ abc \\
\hline $2276 \mathrm{~A} \times 2029 \mathrm{~A}$ & $3,3( \pm 0,33)$ & $3,4( \pm 0,67)$ & $3,4( \pm 0,64)$ & $3,6( \pm 0,40) \mathrm{ab}$ & $4,1( \pm 0,60)$ & $4,1( \pm 0,66)$ & $4,4( \pm 0,52)$ abc & $3,5( \pm 0,22) b c$ & ----- \\
\hline 2029A & $3,2( \pm 0,20)$ & $3,8( \pm 0,47)$ & $4,5( \pm 0,56)$ & $2,6( \pm 0,60) a b$ & $2,8( \pm 0,74)$ & $4,1( \pm 0,59)$ & $3,6( \pm 0,52)$ abc & $4,9( \pm 0,50)$ abc & $5,1( \pm 0,48)$ abc \\
\hline $2029 \mathrm{~A} \times 319 \mathrm{~A}$ & $2,7( \pm 0,30)$ & $3,9( \pm 0,48)$ & $3,8( \pm 0,57)$ & $3,9( \pm 0,53) \mathrm{ab}$ & $5,2( \pm 0,55)$ & $5,6( \pm 0,37)$ & $3,4( \pm 0,58)$ abc & $5,1( \pm 0,48)$ abc & ----- \\
\hline 319A & $2,7( \pm 0,40)$ & $3,1( \pm 0,75)$ & $3,4( \pm 0,75)$ & $2,8( \pm 0,47) a b$ & $3,3( \pm 0,47)$ & $4,5( \pm 0,60)$ & $4,5( \pm 0,75) a b c$ & $5,5( \pm 0,48) b c$ & $4,1( \pm 0,48)$ abc \\
\hline $\boldsymbol{F}$ & 1,41 & 1,03 & 1,30 & 1,74 & 1,21 & 1,53 & 2,87 & 4,17 & 3,66 \\
\hline$p-v a l o r$ & 0,120 & 0,425 & 0,186 & 0,033 & 0,252 & 0,080 & $>0,001$ & $>0,001$ & $>0,001$ \\
\hline
\end{tabular}

Fonte: Autores. 
A não preferência de oviposição poder estar associada a repelentes sintetizados pela planta ou a características físicas da folha (espessura, dureza, pilosidade, cerosidade e textura) que influenciam o comportamento do inseto durante a seleção hospedeira (Vendramin \& Guzzo, 2009; Baldin, Pannuti \& Bentivenha, 2019). A antibiose pode ocorrer devido a produção de metabólitos que prejudicam o desenvolvimento do inseto, como o aumento do período de desenvolvimento larval, redução de peso de larvas e pupas e menor longevidade de adultos (Vendramin \& Guzzo, 2009).

Nesse contexto, o efeito de mecanismos de defesa atuando de modo isolado ou em interação, podem ter contribuído para a redução de danos causados por larvas de $S$. frugiperda. Sendo esse fato expresso pelo baixo nível de danos registrados para todas as variedades crioulas de milho doce avaliadas, independentemente do estádio de desenvolvimento das plantas, pois o nível de ataque pode variar conforme o estágio de desenvolvimento da planta (Siloto, 2002; Moraes, Lourenção \& Paterniani, 2015). Além disso, destaca-se que, por esses fatores afetarem a viabilidade larval, pode ocorrer uma redução significativa no número de indivíduos adultos.

A flutuação populacional de machos de $S$. frugiperda se manteve abaixo do nível de controle (NC) de três mariposas/ha/semana (Cruz, 2015) durante todo o período de avaliação (Figura 1). Picos populacionais de adultos de $S$. frugiperda podem ocorrer entre 30 e 60 dias após a emergência das plantas, momento que se inicia o desenvolvimento do cartucho (Salas, 2001). Nessa fase, variedades suscetíveis apresentam maiores níveis de danos causados por S. frugiperda (Cruz \& Turpin, 1982), sendo ideal para o abrigo e desenvolvimento larval, resultando posteriormente em uma maior captura de adultos.

Figura 1. Flutuação populacional de $S$. frugiperda em vinte variedades de milho doce crioulo. As setas $(\downarrow)$ indicam a aplicação de inseticidas.

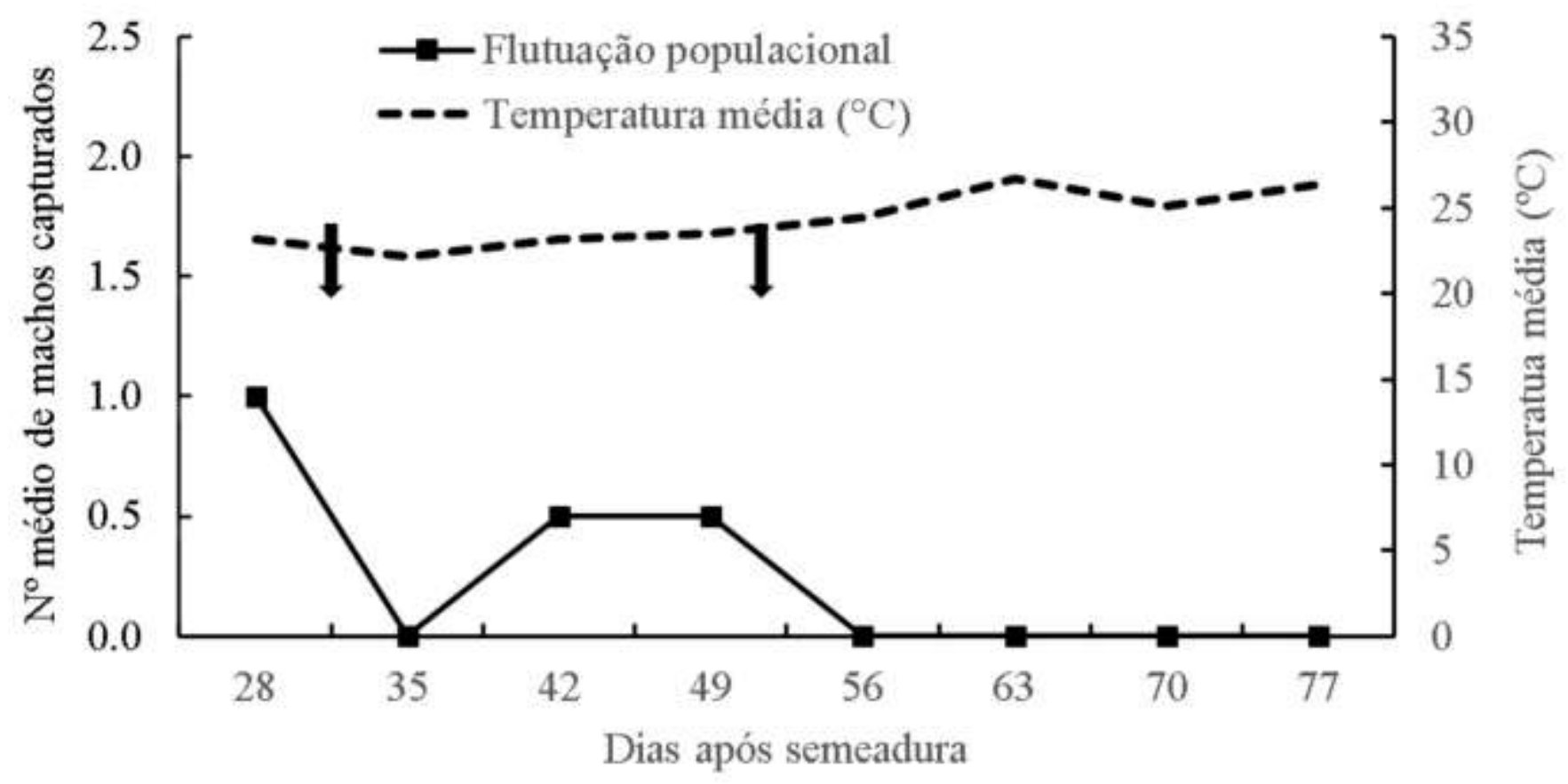

Fonte: Autores.

Entre os fatores que podem ter contribuído para o baixo número de capturas durante o período de amostragem pode estar associado ao uso de inseticidas. Neste contexto, as pulverizações realizadas 34 e 51 DAS foram realizadas observando-se os danos causados por lagartas nas folhas de milho. Entretanto, mesmo com uma reduzida flutuação populacional, houve a ocorrência de danos em plantas. 
A relação entre densidade populacional e nível de dano ainda é objeto de estudo, com trabalhos indicando uma baixa relação entre eles (Girish, 2000; Prasannakumar, Chakravarthy \& Kumar, 2009). Além disso, há uma elevada variabilidade genética em populações de $S$. frugiperda, o que pode comprometer a eficiência de feromônios sexuais sintéticos (Cruz et al., 2012; Pinto et al., 2015), sendo recomendado bioensaios preliminares para verificar a eficácia destes atrativos (Malo et al., 2001). Nesse contexto, o feromônio sintético Bio Spodoptera ${ }^{\circledR}$ empregado neste estudo foi sintetizado a partir de insetos coletados na Costa Rica (Agrofit, 2019), o que pode afetar sua eficiência em locais geograficamente distintos, resultando em correlações negativas entre o nível de dano e a flutuação populacional do inseto. No entanto, destaca-se que o monitoramento é uma estratégia eficaz para estimar a densidade populacional de um inseto, sendo adotado como critério de decisão para o emprego de estratégias de manejo de modo a reduzir a perda de rendimento de uma cultura devido ao ataque de insetos (Cruz et al., 2012).

A diversidade genética existente em milho doce permite a seleção de materiais resistentes ao ataque de pragas, como $S$. frugiperda. O emprego de plantas com essas características mantém insetos praga em baixas densidades, reduzindo o uso de inseticidas, além de ser compatível com outras táticas de manejo. Deste modo, diante dos resultados obtidos, verifica-se a necessidade da realização de mais experimentos a campo e laboratório, avaliando a resistência de genótipos de milho doce ao ataque de $S$. frugiperda, buscando identificar os possíveis materiais resistentes ao ataque de insetos praga.

\section{Considerações Finais}

As variedades de milho doce apresentaram comportamento similar ao ataque de $S$. frugiperda em condições de campo, com danos foliares classificados como baixos (66,7\% das avaliações) e médios (33,3\% das avaliações). O emprego de variedades de milhos resistentes ao ataque de pragas é uma estratégia desejável em programas de manejo integrado de pragas. Nesse contexto, estudos em condições controladas, por meio de infestações artificiais de insetos devem ser realizados, com o objetivo de disponibilizar aos agricultores variedades de milho doce resistentes à $S$. frugiperda. Essas variedades poderão viabilizar a redução no uso de inseticidas químicos sintéticos de amplo espectro, minimizando impactos indesejados ao ambiente e a saúde humana.

\section{Agradecimentos}

Os autores agradecem à Universidade Federal de Santa Catarina (UFSC), à Fazenda Experimental da Ressacada e ao Núcleo de Estudos em Agrobiodiversidade (NEABio).

\section{Referências}

Agrofit. (2019). Sistema de Agrotóxicos Fitossanitários. Ministério da Agricultura, Pecuária e Abastecimento-Coordenação Geral de Agrotóxicos e Afins/DFIA/DAS.

Alvares, C. A., Stape, J. L., Sentelhas, P. C., Gonçalves, J. D. M., \& Sparovek, G. (2013). Köppen's climate classification map for Brazil. Meteorologische Zeitschrift, 22(6), 711-728.

Aoyama, E., \& Labinas, A. (2012). Características estruturais das plantas contra a herbivoria por insetos. Enciclopédia Biosfera, 8(15).

Baldin, E. L. L.; Pannuti, L. E. da R.; Bentivenha, J. P. F. Antixenose. In: Baldin, E. L. L., Vendramim, J. D., \& Lourenção, A. L. (2019). Resistência de plantas a insetos: fundamentos e aplicações. FEALQ, Piracicaba, SP, Brazil.

Barcelos, P. H. S., Angelini, M. R. (2018). Controle de Spodoptera frugiperda (Smith, 1797) em diferentes tecnologias Bts (Bacillus thuringiensis) na cultura do milho. Revista Agricultura Neotropical, 5(1), 35-40.

Bohner, T. O. L., Araújo, L. E. B., \& Nishijima, T. (2013). O impacto ambiental do uso de agrotóxicos no meio ambiente e na saúde dos trabalhadores rurais. Revista eletrônica do curso de direito da UFSM, 8, 329-341.

Bolzan, A. (2019). Monitoramento e caracterização da resistência de Spodoptera frugiperda (JE Smith) (Lepidoptera: Noctuidae) a inseticidas diamidas no Brasil (Doctoral dissertation, Universidade de São Paulo). 
Bordallo, P. D. N., Pereira, M. G., Amaral Júnior, A. T. D., \& Gabriel, A. P. C. (2005). Análise dialélica de genótipos de milho doce e comum para caracteres agronômicos e proteína total. Horticultura Brasileira, 23(1), 123-127.

Casmuz, A., Juárez, M. L., Socías, M. G., Murúa, M. G., Prieto, S., Medina, S., \& Gastaminza, G. (2010). Revisión de los hospederos del gusano cogollero del maíz, Spodoptera frugiperda (Lepidoptera: Noctuidae). Revista de la Sociedad Entomológica Argentina, 69(3-4), 209-231.

Catão, H. C. R. M., Costa, F. M., Valadares, S. V., Dourado, E. D. R., Brandão Junior, D. D. S., \& Sales, N. D. L. P. (2010). Qualidade física, fisiológica e sanitária de sementes de milho crioulo produzidas no norte de Minas Gerais. Ciência Rural, 40(10), $2060-2066$.

Cruz, I., \& Turpin, F. T. (1982). Efeito da Spodoptera frugiperda em diferentes estádios de crescimento da cultura de milho. Pesquisa Agropecuária Brasileira, $17(3), 355-359$

Cruz, I. (1995). A lagarta-do-cartucho na cultura do milho. Embrapa Milho e Sorgo-Circular Técnica (INFOTECA-E).

Cruz, I., Figueiredo, M., \& Da Silva, R. B. (2010). Monitoramento de adultos de Spodoptera frugiperda (JE Smith) (Lepidoptera: Noctuidae) e Diatraea saccharalis (Fabricius) (Lepidoptera: Pyralidae) em algumas regiões produtoras de milho no Brasil. Embrapa Milho e Sorgo-Documentos (INFOTECA-E).

Cruz, I., Figueiredo, M. D. L. C., Silva, R. B. D., Silva, I. F. D., Paula, C. D., \& Foster, J. E. (2012). Using sex pheromone traps in the decision-making process for pesticide application against fall armyworm (Spodoptera frugiperda [Smith] [Lepidoptera: Noctuidae]) larvae in maize. International Journal of Pest Management, 58(1), 83-90.

Cruz, I. (2015). Avanços e desafios no controle biológico com predadores e parasitoides na cultura do milho. https://ainfo.cnptia.embrapa.br/digital/bitstream/item/134910/1/Avancos-desafios.pdf.

Davis, F. M., Williams, W. P., \& Wiseman, B. R. (1989). Methods used to screen maize for and to determine mechanisms of resistance to the Southwestern corn borer and fall armyworm. In International Symposium on Methodologies for Developing Host Plant Resistance to Maize Insects. Mexico, DF (Mexico). 9-14 Mar 1987.

De Andrade, R. V., Dos Santos, M. X., Ferreira, A. D. S., \& De Oliveira, A. C. (2002). Avaliação de acessos de milho crioulo coletados na região central do Brasil. Revista Brasileira de Milho e Sorgo, 1(02).

Diez-Rodríguez, G. I., \& Omoto, C. (2001). Herança da resistência de Spodoptera frugiperda (JE Smith) (Lepidoptera: Noctuidae) a lambda-cialotrina. Neotropical Entomology, 30(2), 311-316.

Fazolin, M., Estrela, J. L. V., Monteiro, A. F. M., Gomes, L. P., Da Silva, I. M., \& Silva, M. D. F. (2015). Sinérgico alternativo para o manejo da resistência da lagarta-do-cartucho do milho a piretróides. Embrapa Acre-Artigo em periódico indexado (ALICE).

Girish, H. M. (2000). Studies on the insects of economic importance attracted to light traps and pheromone traps (Doctoral dissertation, University of Agricultural Sciences Gkvk Bangalore).

Johnson, A. W., Snook, M. E., \& Wiseman, B. R. (2002). Green leaf chemistry of various turfgrasses: Differentiation and resistance to fall armyworm. Crop science, 42(6), 2004-2010.

León-García, I., Rodríguez-Leyva, E., Ortega-Arenas, L. D., \& Solís-Aguilar, J. F. (2012). Susceptibilidad de Spodoptera frugiperda (JE Smith) (Lepidoptera: Noctuidae) a insecticidas asociada a césped en Quintana Roo, México. Agrociencia, 46(3), 279-287.

Malo, E. A., Cruz-Lopez, L., Valle-Mora, J., Virgen, A., Sanchez, J. A., \& Rojas, J. C. (2001). Evaluation of commercial pheromone lures and traps for monitoring male fall armyworm (Lepidoptera: Noctuidae) in the coastal region of Chiapas, Mexico. Florida Entomologist, 659-664.

Moraes, A. R. A. D., Lourenção, A. L., \& Paterniani, M. E. A. G. Z. (2015). Resistência de híbridos de milho convencionais e isogênicos transgênicos a Spodoptera frugiperda (Lepidoptera: Noctuidae). Bragantia, 74(1), 50-57.

Nogueira, L. (2015). Categorias e níveis de resistência de genótipos de milho crioulo a Spodoptera frugiperda (JE Smith, 1797) (Lepidoptera: Noctuidae).

Oliveira Jr, L. F. G., Deliza, R., Bressan-Smith, R., Pereira, M. G., \& Chiquiere, T. B. (2006). Seleção de genótipos de milho mais promissores para o consumo in natura. Food Science and Technology, 26(1), 159-165.

Ota, É. D. C., Lourenção, A. L., Duarte, A. P., Ramos Junior, E. U., \& Ito, M. A. (2011). Desempenho de cultivares de milho em relação à lagarta-do-cartucho. Bragantia, 70(4), 850-859.

Parentoni, S. N., Gama, E. E. G., Magnavaca, R., Reifschneider, F. B., \& Boas, G. L. V. (1990). Milho doce. Embrapa Milho e Sorgo-Artigo em periódico indexado (ALICE).

Pinto, C. C., Grützmacher, A. D., Rosa, A. P. S. A. D., Manica-Berto, R., Mendes, S. M., Arge, L. W. P., \& Borges, C. T. (2015). Diversidade molecular entre populações de Spodoptera frugiperda no Brasil avaliada por marcadores AFLP. Pesquisa Agropecuária Brasileira, 50(4), 343-346.

Prasannakumar, N. R., Chakravarthy, A. K., \& Kumar, L. V. (2009). Relationship between pheromone trap catches and field damage of selected lepidopterous pests on vegetable crops. Pest Management In Horticultural Ecosystems, 15, 63-67.

Ribas, P. P., \& Matsumura, A. T. S. (2009). A química dos agrotóxicos: impacto sobre a saúde e meio ambiente. Revista Liberato, 10(14), 149-158.

Salas, J. (2001). Captura de Spodoptera frugiperda en trampas con feromona.

Dos Santos, H. G., Jacomine, P. K. T., Dos Anjos, L. H. C., De Oliveira, V. A., Lumbreras, J. F., Coelho, M. R., \& Cunha, T. J. F. (2018). Sistema brasileiro de classificação de solos. Brasília, DF: Embrapa, 2018.

Shapiro, S. S., \& Wilk, M. B. (1965). An analysis of variance test for normality (complete samples). Biometrika, 52(3/4), 591-611. 
Research, Society and Development, v. 10, n. 13, e411101321309, 2021

(CC BY 4.0) | ISSN 2525-3409 | DOI: http://dx.doi.org/10.33448/rsd-v10i13.21309

Siloto, R. C. (2002). Danos e biologia de Spodoptera frugiperda (JE Smith, 1797) (Lepidoptera: Noctuidae) em genótipos de milho (Doctoral dissertation, Universidade de São Paulo).

Simmonds, M. S. (2003). Flavonoid-insect interactions: recent advances in our knowledge. Phytochemistry, 64(1), 21-30.

Souza, R. D. (2015). Diversidade de variedades crioulas de milho doce e adocicado conservadas por agricultores do Oeste de Santa Catarina.

Tedesco, J. M.; Gianello, C.; Anghinoni, I.; Bissani, C. A.; Camargo, F. A. O. \& Wiethölter, S. (2004) Manual de Adubação e de Calagem para os estados do Rio Grande do Sul e Santa Catarina.

Teixeira, F. F., De Souza, I. R. P., Gama, E. E. G., Pacheco, C. A. P., Parentoni, S. N., Dos Santos, M. X., \& Meirelles, W. F. (2001). Avaliação da capacidade de combinação entre linhagens de milho doce. Embrapa Milho e Sorgo-Artigo em periódico indexado (ALICE).

Vendramin, J. D.; Guzzo, E. C. Resistência de plantas e a bioecologia e nutrição dos insetos. In: Parra, J. R. P., Panizzi, A. R., Haddad, M. L., Panizzi, A., \& Parra, J. (2009). Bioecologia e nutrição de insetos: base para o manejo integrado de pragas.

Yang, G., Wiseman, B. R., Isenhour, D. J., \& Espelie, K. E. (1993). Chemical and ultrastructural analysis of corn cuticular lipids and their effect on feeding by fall armyworm larvae. Journal of chemical ecology, 19(9), 2055-2074. 